\title{
Hardware Integration and Software Design of Automated Nucleic acid extraction System based on Magnetic Bead Separation Method
}

\author{
Yang $\mathrm{Yu}^{1, \mathrm{a}}$, Yongjie $\mathrm{Dou}^{1, \mathrm{~b}}$, Qian $\mathrm{Li}^{1,2, \mathrm{c}}$, Xiaoli Zhao ${ }^{3}$, Jiansheng $\mathrm{Xu}^{1}$, Congliang \\ Deng $^{3}$, Ming Wang ${ }^{1, d^{*}}$ \\ ${ }^{1}$ Institute of electrical engineering Chinese academy of sciences, Beijing, China \\ ${ }^{2}$ University of Chinese Academy of Sciences, Beijing, China \\ ${ }^{3}$ Plant Laboratory of Beijing Inspection and Quarantine Center, Beijing, China \\ ayuyang@mail.iee.ac.cn, ${ }^{b}$ douyongjie@mail.iee.ac.cn, ${ }^{c}$ liqian@mail.iee.ac.cn,
}

Keywords: nucleic acid extraction; magnetic bead; Plant viral RNA

Abstract. This paper presents a system designed for automated nucleic acid extraction machine based on magnetic bead separation method. A hardware platform has been established which consists of motor controlling module, temperature controlling module and a computer; also, we designed the system software to achieve data acquisition, calculation, display, management and acid extraction control. The sample temperature achieved the required value within $13 \mathrm{~min}$, and the accuracy of temperature control can be up to about \pm 1 degrees Celsius, when the sample amount is the maximum capacity of the sample matrix. And each nucleic acid purification experiment time is controlled within $60 \mathrm{~min}$. Plant viral RNA extraction experiments have been done on this platform. The automatic nucleic acid extraction instrument can be successfully used for the purification of DNA and RNA, and achieved very satisfactory experimental results.

\section{Introduction}

Automated nucleic acid extraction system based on nanobeads is widely used in genomics, molecular diagnostics and medical science [1, 2]. As early as 1999, Hayato Miyachi et al. developed and evaluated an automated specimen preparation instrument for the specific capture of hepatitis $\mathrm{C}$ virus (HCV) RNA with probes and magnetic bead-fluid separation [3]. Subsequently, more and more automated nucleic acid extraction systems were put into use in large laboratories. The use of such platforms for the automation of nucleic acid extraction is a modern approach to handle increasing numbers of samples with limited personnel resources and to minimize the possibility of errors during manual sample handling and preparation [4-10].

At present, there are already some automatic nucleic acid extraction devices in the market, such as Abbott M2000 System [11], Thermo King Fisher Flex [12], and so on. However, these devices have such disadvantages as high using cost, long single purification time et al. considering these, a system for extracting nucleic acids has been developed, and have done some plant viral RNA extraction experiments using this system. The system and the experimental results will be described in detail as follows.

\section{System Realization}

System structure. The architecture of the whole system are shown in Fig 1. It consists of motor controlling module, temperature controlling module and PC. The core of motor controlling module is MPC2810 motion controller based on PC, and this controller is connected to the computer through the computer's PCI bus. The core of temperature controlling module is microcontroller AT89S52.

MPC2810 motion controller receives commands from computer to control four motor drive cards and the stall current detection circuit which controls the crawling and releasing of magnetic head set. Thus, the system can realize a coordinated motion mechanism. The microcontroller controls the system temperature, and communicates with the computer through the serial port. 


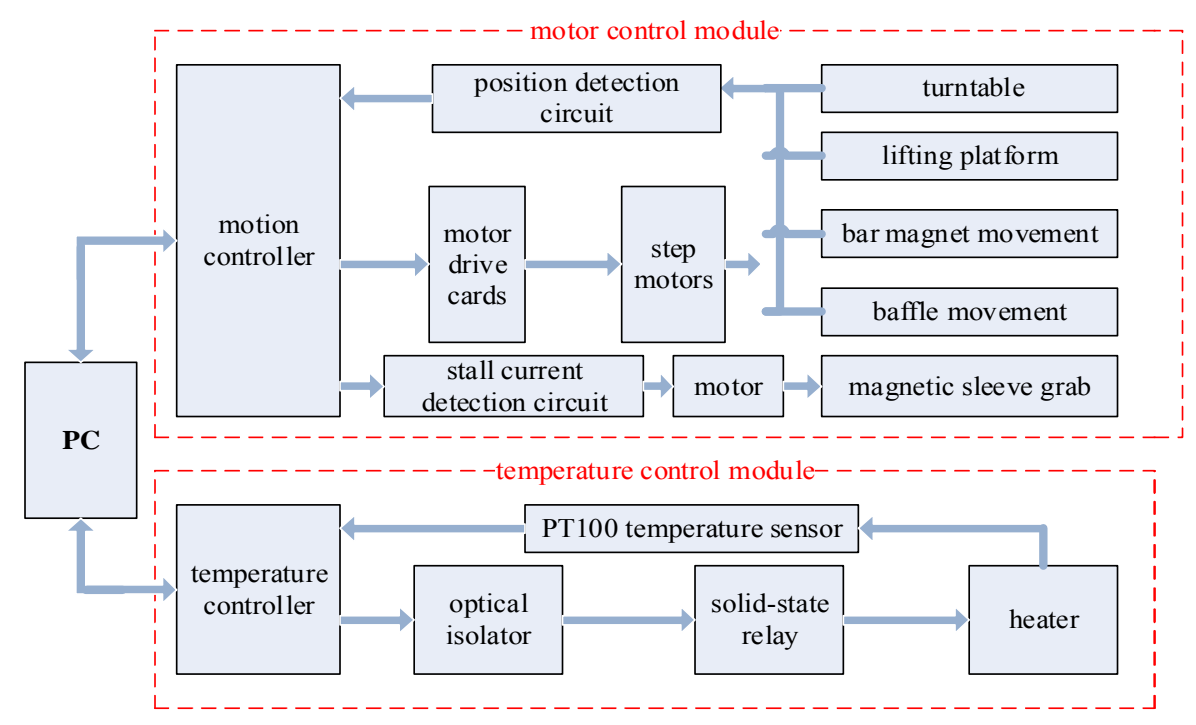

Figure 1. The architecture of the system

Hardware integration. The hardware platform has been implemented and the details of each component will be described as follows:

As shown in Fig. 1, the temperature controlling module involves heater, minimum microcontroller system, PT100 temperature sensor, solid-state relay controlling circuit etc. In this module, PT100 temperature sensor including the signal conditioning circuit is the most important and difficult part, because it directly affects the system temperature resolution and measurement error.

The motor controlling module includes motors, step motor drivers, stall current detection circuit, position detection circuit, MPC2810 motion controller and a PC, as shown in Fig. 1. PC is responsible for management of man-machine interface, and real-time monitoring. The motion controller implements all motion control.

Software design. Software mainly includes PT100 scale transformation formula, microcontroller software and PC software. Each part will be fully described as follows.

PT100 scale transformation formula. Reducing measurement error generally uses look-up table method or mathematical model method. Look-up table method needs to create a large number of data tables, thus need to do many experiments to make multi-point calibration and program versatility is poor. While the program can be very simple and good versatility with mathematical model method.

The system adopts piecewise linear method to reduce measurement error. Firstly, according to the PT100 indexing table, the measurement range was divided into three parts: $0 \sim 49^{\circ} \mathrm{C}, 50 \sim 99^{\circ} \mathrm{C}$, $100 \sim 149^{\circ} \mathrm{C}$. Secondly, the discrete points were linear fitted with least square method, and three equations were obtained:

$$
R=0.388 T+100.02,0^{\circ} C \leq T \leq 49^{\circ} C
$$

$$
R=0.3823 T+100.31,50^{\circ} \mathrm{C} \leq T \leq 99^{\circ} \mathrm{C}
$$

$$
R=0.3764 T+100.88,100^{\circ} C \leq T \leq 149^{\circ} C
$$

In the three mathematical models above, error values are less than $0.1^{\circ} \mathrm{C}$. Using $\mathrm{M}$ and $\mathrm{N}$ as the coefficients of the different temperature ranges, the linear fitting formula is written as Eq. 4.

$$
R=M T+N
$$

$$
\begin{array}{r}
U_{R} A D_{-} \text {Data } / 4096=U \\
\left(R-R_{0}\right) I G=U
\end{array}
$$


$\mathrm{U}_{\mathrm{R}}$ is the reference voltage of an $\mathrm{AD}$ converter, $\mathrm{AD}$-Data is the conversion value of the AD converter, $\mathrm{G}$ is the gain of the amplifying and voltage bias circuit, $\mathrm{R} 0$ is the bias resistor, and $\mathrm{I}$ is the current of the constant current source.

According to the formulas (4), (5), (6), we get the PT100 scale transformation formula:

$$
T=\frac{U_{R} A D_{-} \text {Data }}{4096 I G M}+\frac{R_{0}}{M}-\frac{N}{M}
$$

Microcontroller software. Microcontroller software includes a main program, AD conversion subprogram, digital filtering subprogram, PID control subprogram, and serial communication subprogram. The main program is used to coordinate subprograms to ensure normal operation of the system. The digital filtering subprogram adopted median-value filter to reduce the error and improve the precision of the system. The PID control subprogram utilized an improved segmented PID control algorithm to lower the warming time and improve the stability of the system temperature.

PC software design. PC software was compiled using VB language, mainly includes main interface and parameter setting interface. The GUI is divided into menu bar, control panel area, temperature displaying panel area, and current work progress displaying area. The parameter setting interface is comprised of temperature control parameter setting, solution volume selection, oscillation frequency selection, and position error compensation. In general, the PC software can complete the work of system initialization, real-time display, mode selection, key control, alarming, report printing, et al.

\section{Experiments}

Materials. 1) Sample: The plant leaves with Lily symptomless virus (LSV); 2) Reagents: Reverse Transcriptase M-MLV（RNase H-）（TaKaRa Code：D2639S）, Ribonuclease Inhibitor（TaKaRa Code: D2310A) ,10mM Mixt $\mu$ re dNTP (TaKaRa Code: D4030RA), TaKaRa TaqTM（TaKaRa Code: DR100A）, Premix Ex TaqTM（Perfect real time）（TaKaRa Code: DRR039A）, etc.; 3) Experimental equipment: Automated nucleic acid extraction system we designed, real-time PCR instrument ABI PRISM 7900 Sequence-Detection System, and some other commonly used biological experimental equipment.

Sample preparation. Put a certain amount of plant leaves in a mortar, and add some liquid nitrogen. Then, fully grind using the grinding rod. When the mortar and the pestle sample reach room temperature, an appropriate amount of grinding buffer is added. Grind it evenly and transfer the slurry to $1.5 \mathrm{ml} \mathrm{micro-centrifuge} \mathrm{tube,} \mathrm{and} \mathrm{then} \mathrm{centrifuge} \mathrm{the} \mathrm{tube} \mathrm{at} 5000 \mathrm{rpm}$ for $3 \mathrm{~min}$. Finally, the supernatant was removed and precipitations were diluted 10 -folds serially using the negative control RNA from 0 to 100000 . The samples were used for the automated extraction.

Extraction procedures and automation. In this paper, we use the system we designed to implement the automation of magnetic nanobeads-based extraction method. The system is shown in Fig. 2. Ninety-six samples can be processed in one run. 


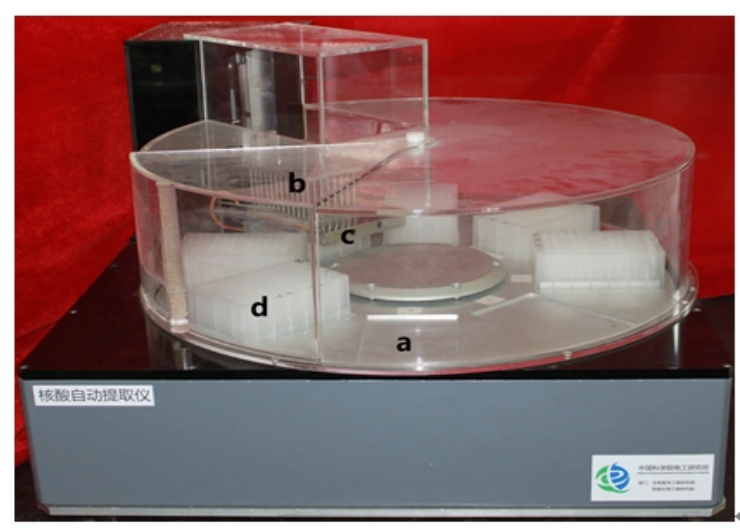

Figure 2. Picture of the system (a) Working station, (b) Magnet array, (c) Magnet set frame, (d) 96-well plate

The system has eight working stations, the first station and the sixth station need to heat, the first station is for the binding of magnetic nanobeads and samples, and the sixth station is for elution. According to the sample purification requirements, two-three stations are for washing, and one station for the magnet set.

Samples, binding buffer, and magnetic nanobeads are put into one 96-well plate. Washing buffer is put into two-three 96-well plates. Elution buffer is put into one 96-well plate. And then put each plate to the corresponding station. Last, start the purification operation. During purification, we mix and collect the samples by magnet up and down movement at different frequencies.

\section{Results and Discussion}

After purification, the plant viral RNA eluate was subjected to RT-PCR assays using the ABI PRISM 7900 Sequence-Detection System according to the manufacturer's instructions.

In this experiment, we did three replicates for each sample. The results are shown in Fig. 3 and Table 1 .

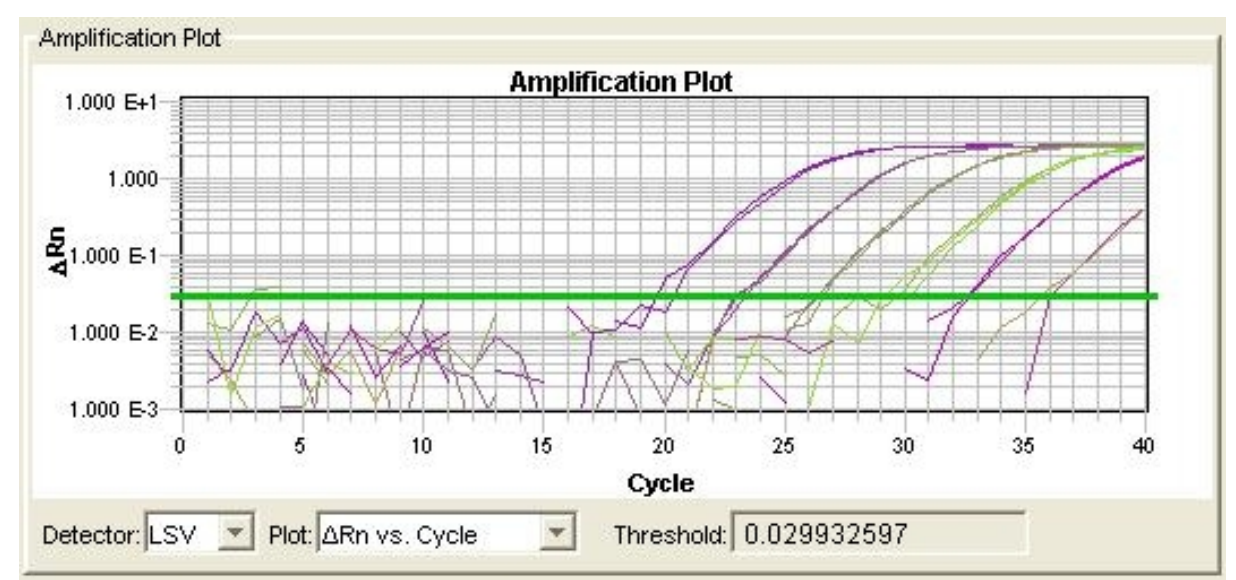

Figure 3. Plant viral RNA amplification curves

Table 1. Ct value of plant viral RNA amplification curves

\begin{tabular}{l|llllll}
\hline Dilution Factor & 0 & $10^{1}$ & $10^{2}$ & $10^{3}$ & $10^{4}$ & $10^{5}$ \\
\hline \multirow{3}{*}{ Ct value } & 20.48957 & 23.28473 & 26.21515 & 29.50733 & 32.66283 & 36.57186 \\
\cline { 2 - 7 } & 19.57528 & 23.43059 & 26.43665 & 30.35444 & 33.763 & 36.27713 \\
\cline { 2 - 7 } & 20.19794 & 23.21688 & 26.55956 & 29.28784 & 33.01285 & 36.60692 \\
\hline Ct average value & 20.0876 & 23.31078 & 26.40379 & 29.71654 & 33.14623 & 36.4835 \\
\hline
\end{tabular}

The concept of the threshold cycle $(\mathrm{Ct})$ is at the heart of accurate and reproducible quantification using fluorescence-based RT-PCR. Fluorescence values are recorded during every cycle and represent the amount of product amplified to that point in the amplification reaction. The more template present at the beginning of the reaction, the fewer number of cycles it takes to reach a point in which the fluorescent signal is first recorded as statistically significant above back-ground. This point is defined 
as the $\mathrm{Ct}$, and will always occur during the exponential phase of amplification [11]. According to the $\mathrm{Ct}$ values, the automated nucleic acid extraction instrument was practical in the extraction of plant viral RNA, and the purification effect and repeatability were very satisfactory.

\section{Conclusions}

In the study, we developed and evaluated a prototype of automated nucleic acid extraction instrument based on magnetic nanobeads. We have used it for the extraction of plant viral RNA, and the extracted RNA was successfully used in an automated PCR assay for the detection of Lily symptomless virus (LSV).

In summary, an automated nucleic acid extraction instrument was developed, and it is suitable for use as a totally automated system, starting with RNA extraction to detection of plant virus, when combined with a fully automated PCR system. In addition to that, it can be used in rapid purification of the protein separation and specific cell separation with special magnetic nanobeads.

\section{Acknowledgements}

This study was financially supported by a Special Fund for the Study of Automatic Detecting for Plant Virus Based on Magnetic Nanobeads (201110035) from General Administration of Quality Supervision, Inspection and Quarantine of the People's Republic of China.

\section{References}

[1] Jun-ichi Akutsu, Yuriko Tojo, Osamu Segawa, Kimimichi Obata, Mina Okochi, Hideji Tajima and Masafumi Yohda. Development of an integrated automation system with a magnetic bead-mediated nucleic acid purification device for genetic analysis and gene manipulation. Biotechnology and Bioengineering, 2004(86): 667-671.

[2] Mathias Bruegel, Mathis Planert, Sven Baumann, Almut Focke, Florian Then Bergh, Alexander Leichtle ,Uta Ceglarek, Joachim Thiery, Georg Martin Fiedler. Standardized peptidome profiling of human cerebrospinal fluid by magnetic bead separation and matrix-assisted laser desorption/ionization time-of-flight mass spectrometry. Journal of Proteomics, 2009(72):608-615.

[3] Hayato Miyachi, Atsuko Masukawa, Toshio Ohshima, Toru Hirose, Chaka Impraim and Yasuhiko Ando 2000 Automated Specific Capture of Hepatitis C Virus RNA with Probes and Paramagnetic Particle Separation J. Clin. Microbiol. 38(1) 18-21

[4] Q. Meng, C. Wong, A. Rangachari, S. Tamatsukuri, M. Sasaki, E. Fiss, L. Cheng, T. Ramankutty, D. Clarke, H. Yawata, Y. Sakakura, T. Hirose and C. Impraim 2001 Automated Multiplex Assay System for Simultaneous Detection of Hepatitis B Virus DNA, Hepatitis C Virus RNA, and Human Immunodeficiency Virus Type 1 RNA J. Clin. Microbiol. 39(8) 2937-2945

[5] M. Nagy, P. Otremba, C. Krüger, S. Bergner-Greiner, P. Anders, B. Henske, M. Prinz, L. Roewer 2005 Optimization and validation of a fully automated silica-coated magnetic beads purification technology in forensics Forensic Science International. 152, 13-22

[6] C.J. Fregeau, C.M. Lett, J. Elliott, C. Yensen, R.M. Fourney, Automated processing of forensic casework samples using robotic workstations equipped with nondisposable tips: contamination prevention, J. Forensic Sci. 53 (2008) 632-651.

[7] Guido Hennig, Mathias Gehrmann,Udo Stropp, Hiltrud Brauch, Peter Fritz, MichelEichelbaum,Matthias Schwab and Werner Schroth 2010 Automated Extraction of DNA and RNA from a Single Formalin-Fixed Paraffin-Embedded Tissue Section for Analysis of Both Single-Nucleotide Polymorphisms and mRNA Expression Clinical Chemistry. 56(12) 1845-1853 
[8] M. Stangegaard, T.G. Frosley, A.J. Frank-Hansen, N. Morling, Automated extraction of DNA from blood and PCR setup using a Tecan Freedom EVO liquid handler for forensic genetic STR typing of reference samples, JALA 16 (2011) 134-140.

[9] W. Tang, M. Zhang, and X. Zeng, "Establishment of dsDNA/GNs/chit/GCE biosensor and electrochemical study on interaction between 6-mercaptopurine and DNA," Bio-medical materials and engineering, vol. 24, pp. 1071-1077, 2014.

[10] H. Su, Z. Su, S. Zheng, H. Yang, and S. Wei, "Interactive cell segmentation based on phase contrast optics," Bio-medical materials and engineering, vol. 24, pp. 29-35, 2014.

[11]http://www.abbottmolecular.com/products/instrumentation-automation/realtime-pcr/m2000-sp-r t.html

[12]http://www.thermoscientific.com/ecomm/servlet/productsdetail?productId=11960640\&groupTy pe $=$ PRODUCT \&searchType $=0 \&$ storeId $=11152 \&$ from $=$ search 